\title{
Lapped Block Image Analysis via the Method of Legendre Moments
}

\author{
Hakim El Fadili \\ Département de physique, Faculté des Sciences Dhar el Mehraz, Université Sidi Mohamed Ben Abdellah, \\ BP 1796 Atlas, Fès, Morocco \\ Email: el_fadili_hakim@hotmail.com

\section{Khalid Zenkouar} \\ Département de physique, Faculté des Sciences Dhar el Mehraz, Université Sidi Mohamed Ben Abdellah, \\ BP 1796 Atlas, Fès, Morocco \\ Email: kzenkouar@hotmail.com

\section{Hassan Qjidaa} \\ Département de physique, Faculté des Sciences Dhar el Mehraz, Université Sidi Mohamed Ben Abdellah, \\ BP 1796 Atlas, Fès, Morocco \\ Email:qjidaa@yahoo.fr
}

Received 22 August 2002 and in revised form 16 April 2003

\begin{abstract}
Research investigating the use of Legendre moments for pattern recognition has been performed in recent years. This field of research remains quite open. This paper proposes a new technique based on block-based reconstruction method (BBRM) using Legendre moments compared with the global reconstruction method (GRM). For alleviating the blocking artifact involved in the processing, we propose a new approach using lapped block-based reconstruction method (LBBRM). For the problem of selecting the optimal number of moment used to represent a given image, we propose the maximum entropy principle (MEP) method. The main motivation of the proposed approaches is to allow fast and efficient reconstruction algorithm, with improvement of the reconstructed images quality. A binary handwritten musical character and multi-gray-level Lena image are used to demonstrate the performance of our algorithm.
\end{abstract}

Keywords and phrases: Legendre moments, global image reconstruction method, block-based reconstruction method, maximum entropy principle, blocking artifact, lapped block-based reconstruction method.

\section{INTRODUCTION}

Moments and functions of moments have been extensively employed as the invariant global features of an image in pattern recognition, image classification, target identification, and scene analysis $[1,2,3,4,5]$.

In the recent years, research investigating the use of moments for pattern reconstruction has been performed. Teh and Chin [6] performed an extensive analysis and comparison of the most common moment definitions, where conventional, Legendre, Zernike, pseudo-Zernike, rotational, and complex moments were all examined in terms of image representation ability, information redundancy, and noise sensitivity. Both analytic and experimental methods were used to characterize the various moment definitions. They concluded that, in terms of overall performances, Zernike and pseudo-Zernike moments outperform the other types.
In general, orthogonal moments are better than other types of moments in terms of information redundancy and image representation.

More recently, an important and significant work considering moments for pattern reconstruction was performed by Liao and Pawlak [7]. In this study, the error analysis and characterization of Legendre moments descriptors have been investigated, where several new techniques to increase the accuracy and the efficiency of the moments are proposed. Based on these improvements, Liao and Pawlak performed image reconstruction via Legendre moments, specially reconstructed image quality.

In order to propose an approach which allows fast and efficient reconstruction algorithm in the case of multi-gray level images with greater sizes, our basic idea is to have high reconstruction quality by using only a small finite number of moments. This strategy lies in the utilization of low-order 
polynomials on small intervals instead of high orders on a single one [8]. Hence, the input image is partitioned into blocks of pixels which are then reconstructed as separate entities. This allows a fast and efficient reconstruction algorithm, with improvement of the reconstructed image quality. Furthermore, if any block is affected by a reconstruction error, the others are not affected, keeping by this way the reconstructed errors as a local distortion; such a fact preserves the integrity of the reconstructed image. Unfortunately, when adjacent blocks have different reconstruction errors, the block boundaries become visible, producing vertical and horizontal lines in the reconstructed images. This blocking artifact is therefore more noticeable at lower reconstruction orders. We propose a new approach for alleviating the blocking artifact using lapped block-based reconstruction method (LBBRM). This method results in significant objective and subjective improvement in image quality.

For the problem of selecting an optimal number of moments from the digital images, Teh and Chin [6] have considered the mean square error (MSE) between an image and its reconstructed version as a good measure of image representation ability. However, this method depends on the unknown original image function, which puts a severe limitation on the application of this criterion. In order to resolve this problem, Liao and Pawlak suggested a statistics cross-validation methodology $[7,9]$. The lack of a complete study concerning this method makes its implementation difficult.

In this paper, we introduce the maximum entropy principle (MEP) as a selection criterion, an automatic technique, which allows estimating the optimal number of moment directly from the available data, where no a priori information is needed $[10,11]$.

Our proposed method, which is the combination of the LBBRM with the MEP as selection criteria, achieves improvement in the four following points:

(i) the reduction of the reconstruction space and, by the way, the reduction of the information quantity to manipulate, involving a great reliability on the reconstruction process where only moments of low orders are used;

(ii) the simplicity of moment calculation and reconstruction into each block constituting the whole image, involving a computation time improvement;

(iii) the robustness against the reconstruction errors which remain local and do not affect the other blocks constituting the whole image;

(iv) the automation of the proposed algorithm, without any a priori information.

In this paper, a class of Legendre orthogonal moments is examined, due to the fact that they possess better reconstruction power than geometrical moments and they attain a zero value of redundancy measures $[6,12,13,14]$. Nevertheless, the presented results can be extended to other types of orthogonal moments $[6,14,15,16]$.
Our paper is organized as follows. In Section 2, some basic definitions are given to build up the necessary mathematical background, including Legendre moments and their properties. Section 3 performs the block-based reconstruction method (BBRM) using the MEP. Section 4 presents the LBBRM method and its performance. Finally, Sections 5 and 6 deal with the summary of important results and conclusions of the paper.

\section{LEGENDRE MOMENTS}

The Legendre moments of order $(p+q)$ are defined for a given real image intensity function $f(x, y)$ as

$$
\lambda_{p, q}=\frac{(2 p+1)(2 q+1)}{4} \int_{R} \int_{R} P_{p}(x) P_{q}(y) f(x, y) d x d y,
$$

where $f(x, y)$ is assumed to have bounded support.

The Legendre polynomials $P_{p}(x)$ are a complete orthogonal basis set on the interval $[-1,1]$, for an order $p$. They are defined as

$$
P_{p}(x)=\frac{1}{2^{p} p !} \frac{d^{p}}{d x^{p}}\left(x^{2}-1\right)^{p} .
$$

The orthogonality property is guaranteed by the equality

$$
\int_{-1}^{1} P_{p}(x) P_{q}(x) d x=\frac{2}{(2 p+1)} \delta_{p, q}
$$

where $\delta_{p, q}$ is the Kronecker function, that is,

$$
\delta_{p, q}= \begin{cases}1 & \text { if } p=q \\ 0 & \text { otherwise. }\end{cases}
$$

\subsection{Image reconstruction by Legendre moments}

By taking the orthogonality principle into consideration, the image function $f(x, y)$ can be written as an infinite series expansion in terms of the Legendre polynomials over the square $[-1,1] \times[-1,1]$ :

$$
f(x, y)=\sum_{p=0}^{\infty} \sum_{q=0}^{\infty} \lambda_{p, q} P_{p}(x) P_{q}(y),
$$

where the Legendre moments $\lambda_{p, q}$ are computed over the same square.

If only Legendre moments of order smaller than or equal to $\theta$ are given, then the function $f(x, y)$ can be approximated by a continuous function which is a truncated series:

$$
f_{\theta}(x, y)=\sum_{p=0}^{\theta} \sum_{q=0}^{p} \lambda_{p-q} P_{p-q}(x) P_{q}(y) .
$$

Furthermore, $\lambda_{p, q}$ 's must be replaced by their numerical approximation which will be pointed out in the following 
section. The number of moments used in the reconstruction of the image for a given order $\theta$ is defined by

$$
N_{\text {total }}=\frac{(\theta+1)(\theta+2)}{2} .
$$

\subsection{Approximation of the Legendre moments}

The aforementioned properties of the Legendre moments are valid as long as one uses a true analog image function. In practice, the Legendre moments have to be computed from sampled data, that is, the rectangular sampling of the original image function $f(x, y)$, producing the set of samples $f\left(x_{i}, y_{j}\right)$ with an $(M, N)$ array of pixels; thus we define the discrete version of $\lambda_{p, q}$ in terms of summation by the traditional commonly used formula (see [6]):

$$
\tilde{\lambda}_{p, q}=\frac{(2 p+1)(2 q+1)}{4} \sum_{i=1}^{M} \sum_{j=1}^{N} P_{p}\left(x_{i}\right) P_{q}\left(y_{j}\right) f\left(x_{i}, y_{j}\right) \Delta x \Delta y,
$$

where $\Delta x=\left(x_{i}-x_{i-1}\right)$ and $\Delta y=\left(y_{j}-y_{j-1}\right)$ are sampling intervals in the $x$ and $y$ directions.

It is clear, however, that $\tilde{\lambda}_{p, q}$ is not a very accurate approximation of $\lambda_{p, q}$, in particular, when the moment order $(p+q)$ increases [7].

The piecewise constant approximation of $f(x, y)$ in $(1)$, proposed recently by Liao and Pawlak [7, 9], yields the following approximation of $\lambda_{p, q}$ :

$$
\hat{\lambda}_{p, q}=\sum_{i=1}^{M} \sum_{j=1}^{N} H_{p, q}\left(x_{i}, y_{j}\right) f\left(x_{i}, y_{j}\right)
$$

with the supposition that $f(x, y)$ is piecewise constant over the interval

$$
\left[x_{i}-\frac{\Delta x}{2}, x_{i}+\frac{\Delta x}{2}\right] \times\left[y_{j}-\frac{\Delta y}{2}, y_{j}+\frac{\Delta y}{2}\right],
$$

and where

$$
\begin{aligned}
& H_{p, q}\left(x_{i}, y_{j}\right) \\
& \quad=\frac{(2 p+1)(2 q+1)}{4} \int_{x_{i}-\Delta x / 2}^{x_{i}+\Delta x / 2} \int_{y_{j}-\Delta y / 2}^{y_{j}+\Delta y / 2} P_{p}(x) P_{q}(y) d x d y
\end{aligned}
$$

represents the integration of the polynomial $P_{p}(x) P_{q}(y)$ around the $\left(x_{i}, y_{j}\right)$ pixel.

This approximation allows a good quality of reconstructed images by reducing the reconstruction error. But, in this study, only bilevel and small-size images are taken into account, that is, multi-gray-level images with greater sizes have been ignored. Indeed, if we consider those later, higherorder moments are involved, and by the way, the computation of moments becomes a time-consuming procedure, too long to be tolerated, with no high quality image successfully reconstructed from the original version.

\section{BLOCK IMAGE RECONSTRUCTION}

To overcome this situation, our strategy lies in the utilization of polynomials having low orders on small intervals instead of high orders on a single one [8]; that is, the input image is partitioned into square blocks of pixels of size $(k, l)$, a thing that produces a number of subimages which will be reconstructed separately.

Let $(M, N)$ be the image size by pixels and let $(k, l)$ represent the block size. By introducing the variables

$$
s_{1}=\frac{M}{k}, \quad s_{2}=\frac{N}{l},
$$

we can deduce the total number of image blocks, which can be set as $N_{b}=s_{1} \cdot s_{2}$.

Given the image space which takes the form

$$
\Omega=\left\{x_{i}, y_{j} \mid 0 \leq x_{i} \leq M, 0 \leq y_{j} \leq N\right\},
$$

we define the subset $D^{n_{1}, n_{2}} \subset \Omega$ as

$$
\begin{aligned}
& D^{n_{1}, n_{2}}=\left\{x_{i}, y_{j} \mid n_{2} k\right. \leq x_{i} \leq\left(n_{2}+1\right) k, \\
&\left.n_{1} l \leq y_{j} \leq\left(n_{1}+1\right) l\right\} .
\end{aligned}
$$

It should be noticed that this subspace, which can also be termed image block space (see Figure 1), is related to $\Omega$ with

$$
\Omega=\bigcup_{n_{1}=0}^{\left(s_{1}-1\right)} \bigcup_{n_{2}=0}^{\left(s_{2}-1\right)} D^{n_{1}, n_{2}} .
$$

Then let the image function associated to each $D^{n_{1}, n_{2}}$ subset be defined as follows:

$$
f^{n_{1}, n_{2}}(x, y)=\left\{f\left(x_{i}, y_{j}\right) \mid x_{i}, y_{j} \in D^{n_{1}, n_{2}}\right\} .
$$

This gives

$$
f(x, y)=\bigcup_{n_{1}=0}^{\left(s_{1}-1\right)} \bigcup_{n_{2}=0}^{\left(s_{2}-1\right)} f^{n_{1}, n_{2}}(x, y)
$$

From these definitions, we introduce the Legendre moment related to each image block as

$$
\hat{\lambda}_{p, q}^{n_{1}, n_{2}}=\sum_{i=n_{2} k}^{\left(n_{2}+1\right) k} \sum_{j=n_{1} l}^{\left(n_{1}+1\right) l} H_{p, q}^{n_{1}, n_{2}}\left(x_{i}, y_{j}\right) f^{n_{1}, n_{2}}\left(x_{i}, y_{j}\right),
$$

where

$$
H_{p, q}^{n_{1}, n_{2}}\left(x_{i}, y_{j}\right)=\int_{x_{i}-\Delta x / 2}^{x_{i}+\Delta x / 2} \int_{y_{j}-\Delta y / 2}^{y_{j}+\Delta y / 2} P_{p}(x) P_{q}(y) d x d y
$$

and $x_{i}, y_{j} \in D^{n_{1}, n_{2}}$. 


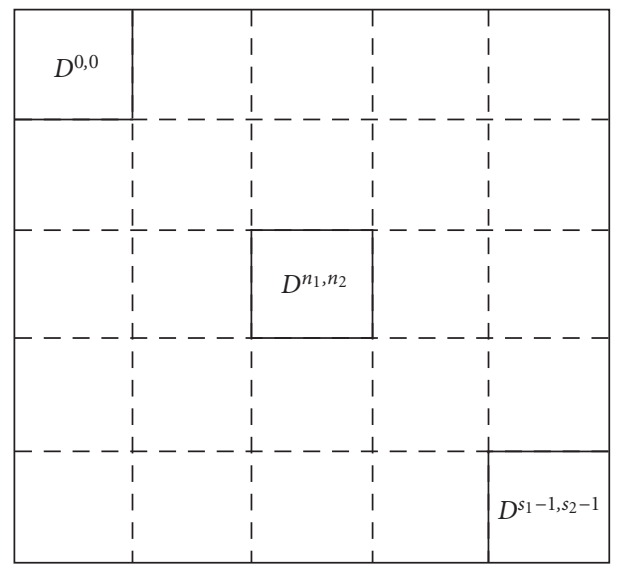

(a)

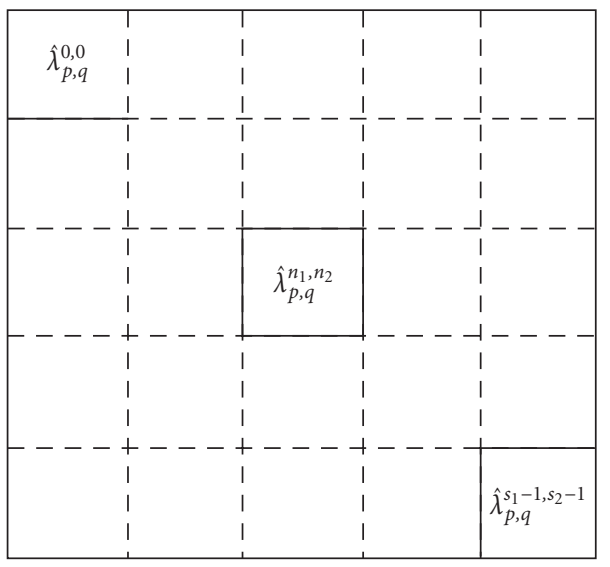

(b)

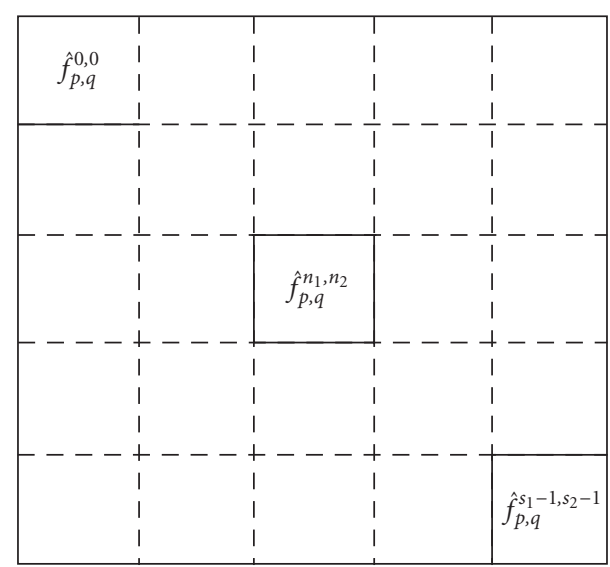

(c)

FIGURE 1: Illustration of the BBRM. (a) Division of the input image into $N_{b}$ subimages, (b) moment extraction for each block, and (c) reconstruction and merging the $N_{b}$ blocks.
The block image function reconstructed from $\lambda_{p, q}^{n_{1}, n_{2}}$ up to a given order $\theta$ can intuitively be defined as

$$
\hat{f}_{\theta}^{n_{1}, n_{2}}\left(x_{i}, y_{j}\right)=\sum_{p}^{\theta} \sum_{q}^{m} \hat{\lambda}_{p-q, q}^{n_{1}, n_{2}} P_{p-q}\left(x_{i}\right) P_{q}\left(y_{j}\right) .
$$

The image function up to $\theta$ can be finally obtained by

$$
\hat{f}_{\theta}(x, y)=\bigcup_{n_{1}=0}^{s_{1}-1} \bigcup_{n_{2}=0}^{s_{2}-1} \hat{f}_{\theta}^{n_{1}, n_{2}}(x, y) .
$$

The proposed technique of reconstruction achieves the first three improvements presented in the introduction.

\subsection{Optimal-order moments selection using MEP}

The image recovery from its moments is quite difficult and computationally expansive because we ignore the order of the truncated expansion of $f(x, y)$, which gives a good quality of the reconstructed image.

Here, we introduce the MEP for the reconstruction. This automatic technique can estimate the optimal number of moments directly from the available data and does not require any a priori image information.

Let $\hat{p}\left(x_{i}, y_{j}\right)$ be the estimated probability density function obtained by normalizing $\hat{f}\left(x_{i}, y_{j}\right)[10]$ :

$$
\hat{p}\left(x_{i}, y_{j}\right)=\frac{\hat{f}\left(x_{i}, y_{j}\right)}{\sum_{x_{i}, y_{j} \in \Omega} \hat{f}\left(x_{i}, y_{j}\right)},
$$

with

$$
\sum_{x_{i}, y_{j} \in \Omega} \hat{p}\left(x_{i}, y_{j}\right)=1
$$

where $0 \leq \hat{p}\left(x_{i}, y_{j}\right) \leq 1$, and $\Omega$ is the image plane.

Let $G_{w}$ be a set of estimated underlying probability density function for various Legendre moment orders $\theta$ :

$$
G_{w}=\left\{\hat{p}_{\theta}\left(x_{i}, y_{j}\right) \mid \theta=1, \ldots, \omega\right\} .
$$

By applying the MEP for noisy images, we deduce that among these estimates of the probability density function, there is one and only one probability density function denoted as $\hat{p}_{\theta}^{*}\left(x_{i}, y_{j}\right)$ whose entropy is maximum $[10,17]$ and which represents the optimal probability density function, and then gives the optimal order of moments. For noise-free images, the entropy function monotonically increases up to a certain optimal order where the maximum image information is recreated, and then become relatively constant.

The Shannon entropy of $\hat{p}_{\theta}^{*}\left(x_{i}, y_{j}\right)$ is defined as in [11]:

$$
S\left(\hat{p}_{\theta}\left(x_{i}, y_{j}\right)\right)=-\sum_{x_{i}, y_{j} \in \Omega} \hat{p}_{\theta}\left(x_{i}, y_{j}\right) \log \left(\hat{p}_{\theta}\left(x_{i}, y_{j}\right)\right)
$$


and the optimal $\hat{p}_{\theta}^{*}\left(x_{i}, y_{j}\right)$ is such that

$$
S\left(\hat{p}_{\theta}^{*}\left(x_{i}, y_{j}\right)\right)=\max \left\{S\left(\hat{p}_{\theta}\left(x_{i}, y_{j}\right)\right) \mid \hat{p}_{\theta}\left(x_{i}, y_{j}\right) \in G_{w}\right\} .
$$

\subsection{Block reconstruction algorithm using the MEP}

The Legendre moments representation and reconstruction method by block processing is the same as illustrated in Section 2, except that, in this case, the algorithm will try to reconstruct each block separately; and the optimal order of reconstruction, controlled by the MEP, will be given after merging all the subimages into the whole output image.

The following are the steps of the block reconstruction algorithm using the MEP as a measure of moment-order selection; here we use the following iterative algorithm version:

Initialize $\theta$.

Divide the original image into square blocks of size $(k \times l)$.

Repeat

(1) Increase $\theta$.

(2) Evaluate the Legendre moments of each block by using (18).

(3) Estimate the image density function of each block by using (20).

(4) Merge the estimated blocks into the whole image $\hat{f}_{\theta}\left(x_{i}, y_{j}\right)$ for the order $\theta$ using (21).

(5) Evaluate the corresponding Shannon entropy $S\left(\hat{p}_{\Theta}\left(x_{i}, y_{j}\right)\right)$.

Until $S\left(\hat{p}_{\theta}\left(x_{i}, y_{j}\right)\right) \leq S\left(\hat{p}_{\theta}\left(x_{i}, y_{j}\right)\right)+\varepsilon$.

Algorithm 1

Take $\theta$ as the optimal order and $\hat{f}_{\theta}\left(x_{i}, y_{j}\right)$ as the optimal reconstructed density function.

To evaluate experimentally the values taken by $\varepsilon$, computer simulations have been carried out, applied on Lena and handwritten musical character with different input block sizes $(4 \times 4),(8 \times 8),(16 \times 16)$, and $(32 \times 32)$.

The iterative LBBRM algorithm produces good results in terms of image quality only if $\varepsilon$ verifies the following condition:

$$
|\varepsilon|<1 \text {. }
$$

The values of $\varepsilon$ according to the results indicated in Table 1 are indicated in Section 5.

\section{LAPPED BLOCK-BASED RECONSTRUCTION METHOD}

The proposed block image reconstruction using the method of moments offers a good trade-off between computation
TABLE 1: The optimal orders given by the MEP in each block size of the LBBRM and GRM, with the corresponding image qualities.

\begin{tabular}{lccccc}
\hline & \multicolumn{4}{c}{ LBBRM } & GRM \\
\cline { 2 - 5 } Block size & $4 \times 4$ & $8 \times 8$ & $16 \times 16$ & $32 \times 32$ & \\
Optimal order & 4 & 8 & 13 & 25 & 80 \\
PSNR (dB) & 32.67 & 36.19 & 29.20 & 26.12 & 24.08 \\
\hline
\end{tabular}

time and subjective image quality. Unfortunately, when adjacent blocks have different reconstruction errors, the block boundaries become visible. This blocking artifact is therefore more noticeable in the reconstructed images at lower reconstruction orders. These vertical and horizontal lines, caused by this blocking artifact, are generally considered objectionable to human viewers.

One technique for mitigating artifacts in block processing involves a posteriori processing of the reconstructed images. Such techniques allow substantial reduction of the blocking artifact, despite the expense of an increase in the overall mean square reconstruction error [18].

It is well known that the blocking effect is a consequence of ignoring the interblock correlation during the reconstruction process because every block is taken as an independent entity. Therefore, one of the best ways to minimize the disturbance in the output image is to make use of the interblock correlation. Our method exploits for every block the neighborhood information related to its adjacent blocks during the moment computation. This approach can achieve a remarkable performance in eliminating the blocking effect and, by the way, avoid other strategies to restore or enhance the image quality by using postprocessing techniques. Consequently, the elimination of the blocking artifact is included in the moment computation and reconstruction process.

Figure 2 shows the block diagram of the proposed LBBRM. Note that there are two stages in this block diagram:

(i) the moment computation which extracts the block neighborhood information by proceeding on lapped blocks;

(ii) the reconstruction process which acts on output blocks and merge them into the final image.

The proposed LBBRM algorithm controlled by the entropy principle is the same as in the previous section, except that moments are computed for lapped blocks composing the input image as defined in (18), and according to Figure 2, the image function is obtained by merging the output blocks as defined in (20) and (21).

Controlled by the entropy principle, the LBBRM approach estimates the optimal number of moments directly from the available data. Consequently, it does not require any a priori information about the image.

As a summary, the LBBRM achieves improvement in the following points:

(i) mitigating the artifact involved in the block processing by exploiting the block neighborhood information 


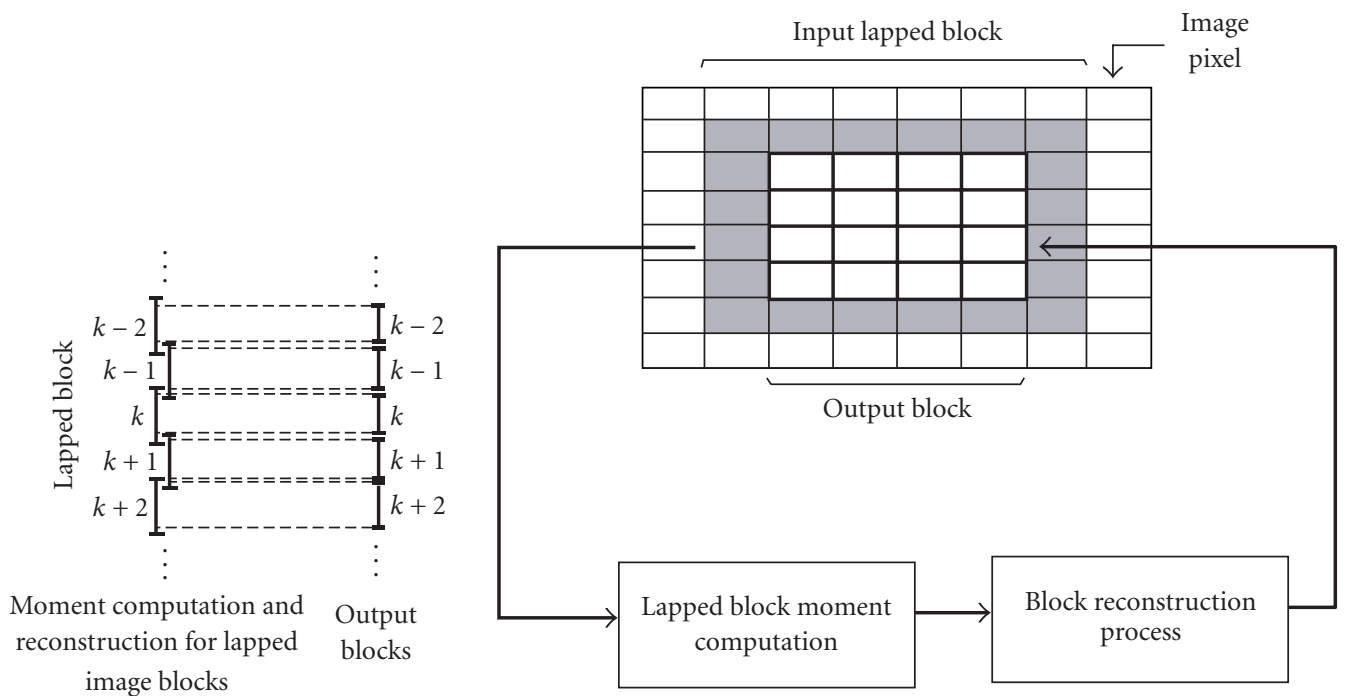

FIGURE 2: Illustration of the different stages of the LBBRM using Legendre moments.

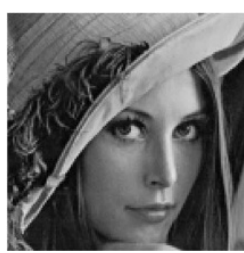

(a)

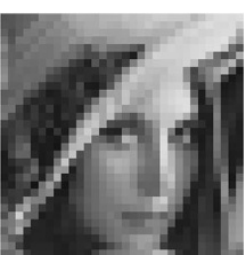

PSNR $=16.23$

(b)

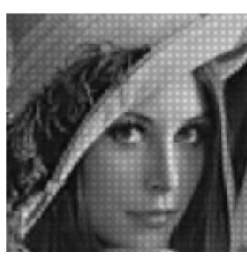

PSNR $=22.55$

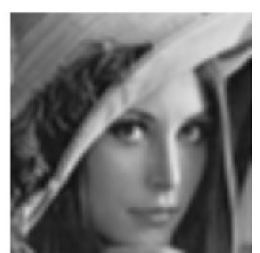

$\operatorname{PSNR}=23.12$

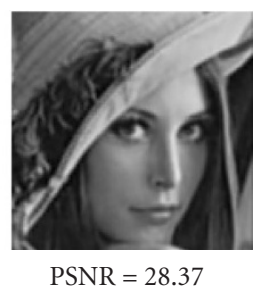

(c)

Figure 3: (a) The original Lena image, (b) the reconstructed Lena via the BBRM (left image) and LBBRM (right image) using Legendre moments with the block size $(4 \times 4)$ and for reconstruction order 0 , and (c) the image via the BBRM (left image) and LBBRM (right image) for block size $(4 \times 4)$ and order 4 . during the moment computation stage, a thing that allows to avoid enhancement postprocessing techniques which is a time-consuming procedure.

(ii) the automatic estimation of the optimal order from the available data; hence, there is no need to a priori information about image.

As shown in Figure 3, this method results in significant objective and subjective improvement in image quality.

\section{EXPERIMENTAL RESULTS}

In this section, we introduce some criteria commonly used for measuring image quality and, therefore, rating the performance of the reconstruction as a processing technique.

\subsection{Mean square error}

The MSE is defined for an image having the size $(M, N)$ as

$$
\text { MSE }=\frac{1}{M N} \sum_{i=1}^{M} \sum_{j=1}^{N}\left|f\left(x_{i}, y_{j}\right)-\hat{f}\left(x_{i}, y_{j}\right)\right|^{2},
$$

where $\hat{f}\left(x_{i}, y_{j}\right)$ is the reconstructed version of the original function $f\left(x_{i}, y_{j}\right)$ over the $\left(x_{i}, y_{j}\right)$ pixel.

\subsection{Peak signal-to-noise ratio}

In [19], the peak signal-to-noise ratio (PSNR) is defined in decibels $(\mathrm{dB})$ as

$$
\mathrm{PSNR}=10 \log _{10}\left(\frac{k^{2}}{\mathrm{MSE}}\right)
$$




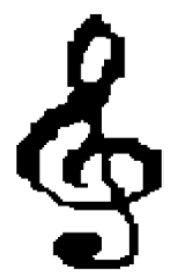

(a)

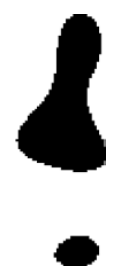

(b)

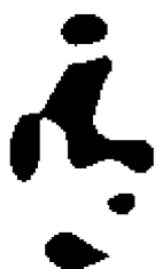

(c)

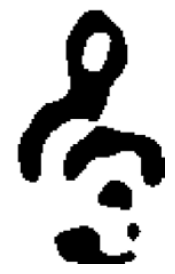

(d)

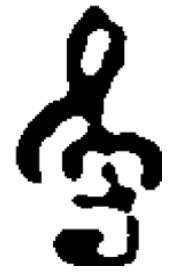

(e)

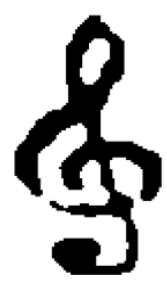

(f)

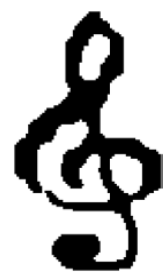

(g)

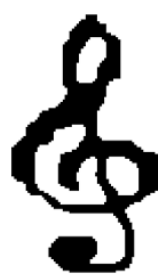

(h)

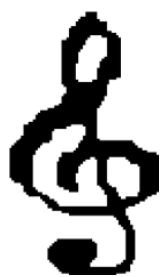

(i)

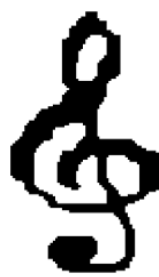

(j)

FIgURE 4: Global reconstruction of the "Clef de Sol" image by the Legendre moments. (a) Original input image; reconstructed images from (b) to (j) represent orders $10,20,30,40,50,60,70,80$, and 90, respectively.

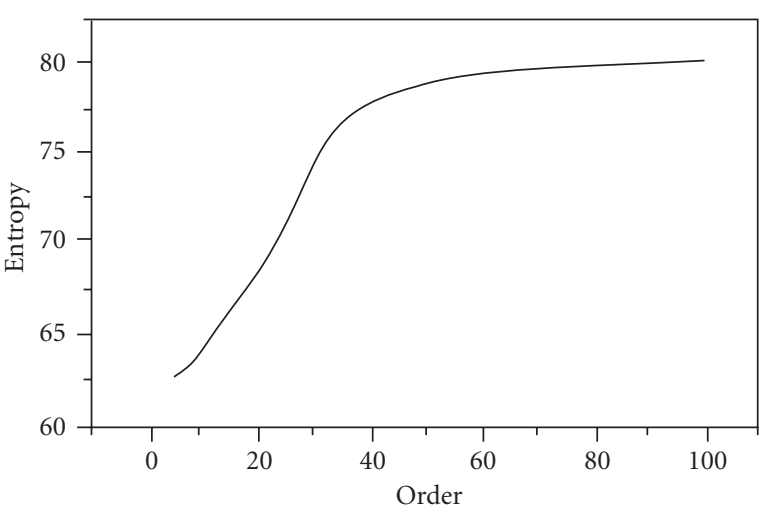

FIGURE 5: The corresponding entropy function of the reconstructed images versus the order of reconstruction. The optimal order of the reconstruction is 80, with PSNR 24.08.

where $k$ is the gray-level maximum value of the original image.

In order to illustrate our approach, we consider a $(128 \times$ 128) real binary image representing a special handwritten musical note called "Clef de Sol" scanned at 256 gray-level, and binarized into 0 and 255 (Figure $4 \mathrm{a})$, and the $(128 \times 128)$ real multi-gray-level Lena image.

Figures 4 and 9 show examples of the reconstruction for the $(128 \times 128)$ handwritten character and Lena image, respectively, from its Legendre moment with the classical global reconstruction method (GRM), where the processing is done on the whole image by including increasingly higherorder moments. It illustrates the fact that the fine detail can

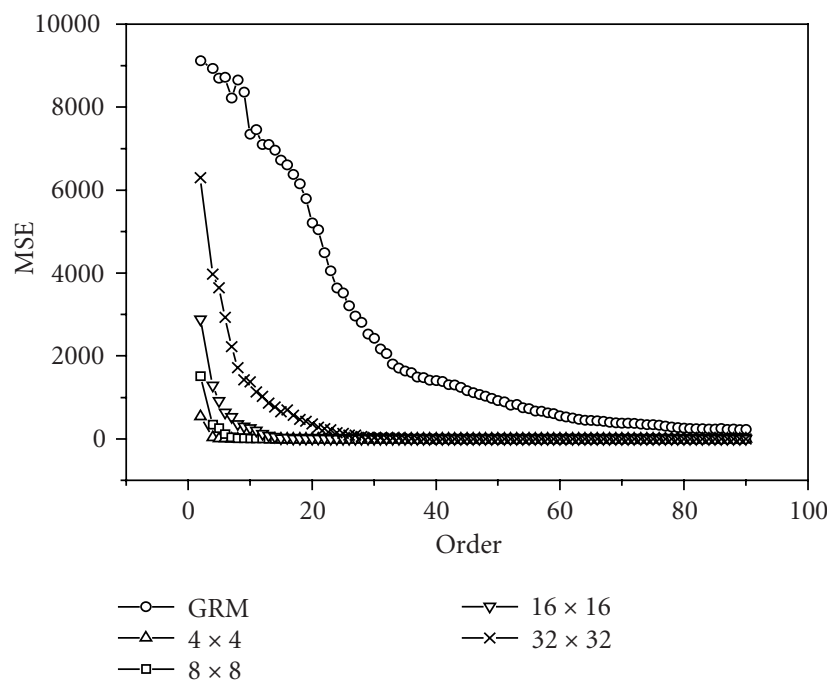

FIgure 6: Comparison of the LBBRM, with different block sizes, and GRM in terms of the reconstruction MSE for the handwritten musical character.

be recreated only by including higher-order moments. The corresponding entropy function of the handwritten character, as defined in (25), is computed and shown versus the reconstruction order in Figure 5.

The reconstructed images for the same handwritten character and the Lena image by the LBBRM, including sizes $(4 \times 4)$ and $(8 \times 8)$, are shown in Figures 7,10 , and 11 , respectively. It shows that a relatively small finite set of moments can adequately characterize the given image 


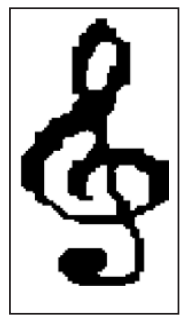

(A)

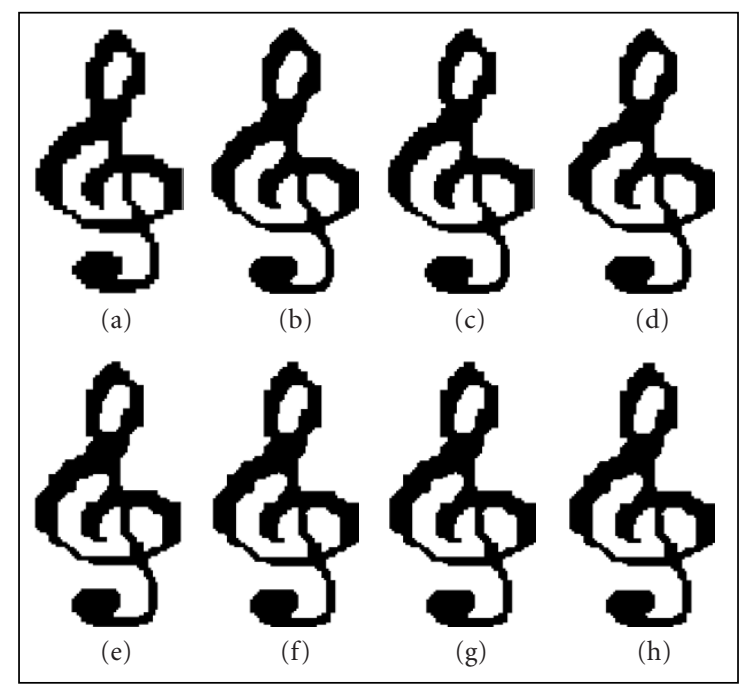

(B)

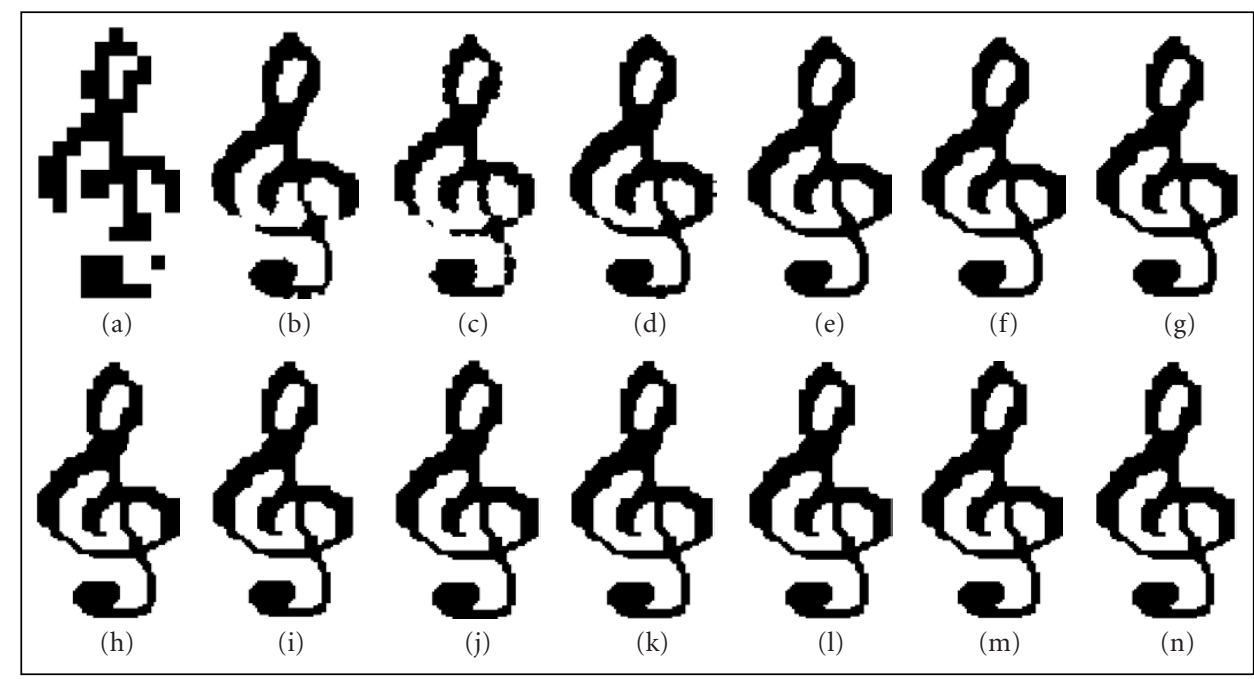

(C)

FIGURE 7: The original image representing the handwritten musical character "Clef de Sol," and its reconstructed pattern via the LBBRM using Legendre moments. (A) The original input image. (B) LBBRM with the block size $(4 \times 4)$; from $(\mathrm{a})$ to $(\mathrm{g})$ the reconstructed images from the order 0 to $6,(\mathrm{~h})$ the reconstructed image for the order 7 where the reconstruction error is equal to zero. (C) LBBRM with the block size reconstruction $(8 \times 8)$, from $(\mathrm{a})$ to $(\mathrm{m})$ the reconstructed images from the order 1 to 12 , (n) reconstructed image for the order 13 where the perfect reconstruction is obtained. 


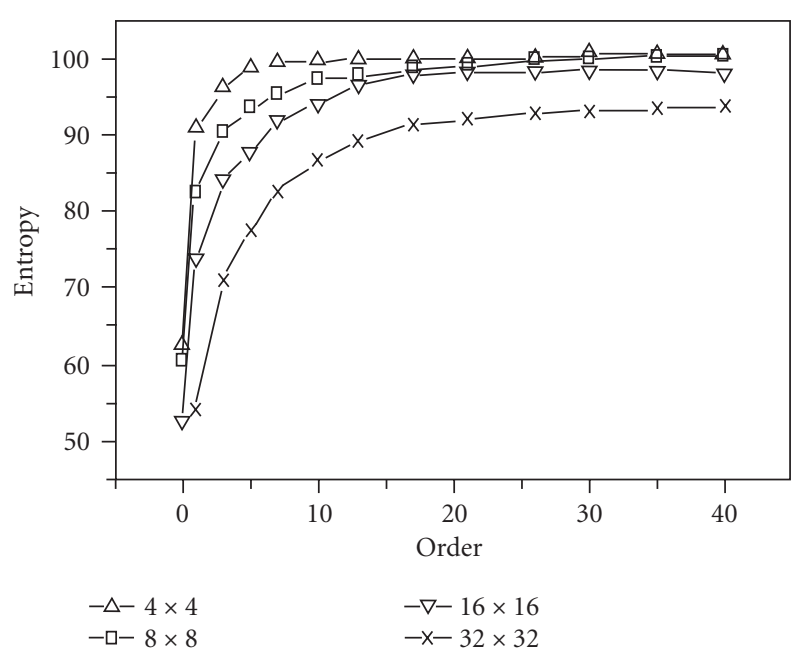

FIGURE 8: Entropy function for the reconstructed images via the new proposed LBBRM with different sizes.

with no need to include higher-order moments. The corresponding entropy functions in each case are regrouped in Figure 8.

Table 1 summarizes the optimal orders obtained in each case with the corresponding PSNR. The following values of $\varepsilon$ have been obtained according to the results indicated in Table 1:

(i) for $(4 \times 4)$ block size, $\varepsilon=0.70$ with optimal order $=4$;

(ii) for $(8 \times 8)$ block size, $\varepsilon=0.85$ with optimal order $=8$;

(iii) for $(16 \times 16)$ block size, $\varepsilon=0.91$ with optimal order $=13$;

(iv) for $(32 \times 32)$ block size, $\varepsilon=0.93$ with optimal order $=25$.

It is clear from the results that the optimal number of moments, which are useful to the reconstruction process, increases as the reconstruction block size increases. The comparison in terms of the mean square reconstruction errors (MSE), as defined in (28), of GRM and LBBRM, is plotted in Figure 6 for the handwritten character, and in Figure 12 for the Lena image.

Figures 6 and 12 show the dramatic reduction of the reconstruction error while reconstructing image by smaller block sizes. As shown in Table 2, the proposed LBBRM can recreate high-quality images for lower-order of moments, compared with the GRM. Table 3 shows the important computation time-reduction factors obtained by using the proposed LBBRM compared with the GRM.

\section{CONCLUSION}

This paper proposes a new technique based on block image reconstruction using Legendre moments. We propose, for the problem of selecting the optimal number of mo-
TABLE 2: Values of the PSNR ( $\mathrm{dB}$ ) for the reconstructed images representing Lena for the proposed LBBRM, with different block sizes, and GRM via the reconstruction order.

\begin{tabular}{rrrrrr}
\hline \multicolumn{5}{c}{ PSNR with LBBRM } & PSNR with GRM \\
\cline { 3 - 5 } Size & $4 \times 4$ & $8 \times 8$ & $16 \times 16$ & $32 \times 32$ & \\
\cline { 1 - 3 } Order & & & & & \\
\cline { 1 - 3 } 0 & 21.91 & 18.56 & 15.96 & 13.45 & 11.21 \\
5 & 28.98 & 22.62 & 21.72 & 19.55 & 16.24 \\
10 & 35.32 & 25.36 & 24.86 & 22.15 & 17.42 \\
15 & 36.22 & 34.38 & 25.95 & 24.12 & 18.32 \\
20 & 37.11 & 34.81 & 27.70 & 25.59 & 19.34 \\
25 & 38.94 & 31.91 & 32.95 & 26.85 & 20.35 \\
30 & 35.22 & 32.68 & 33.64 & 28.25 & 21.19 \\
35 & 38.00 & 36.91 & 35.83 & 29.58 & 21.89 \\
40 & 39.24 & 38.64 & 37.47 & 31.16 & 22.70 \\
45 & 41.77 & 38.23 & 35.18 & 32.56 & 23.28 \\
50 & 40.38 & 39.52 & 33.66 & 33.54 & 23.66 \\
\hline
\end{tabular}

TABLE 3: The reduction factors of reconstruction time for LBBRM in comparison with GRM for PSNR = 26.

\begin{tabular}{lcccc}
\hline & \multicolumn{4}{c}{ LBBRM } \\
\cline { 2 - 5 } Block size & $4 \times 4$ & $8 \times 8$ & $16 \times 16$ & $32 \times 32$ \\
\hline Musical character & $80.23 \%$ & $86.61 \%$ & $86.45 \%$ & $81.66 \%$ \\
Corresponding moment order & 1 & 3 & 6 & 13 \\
\hline Lena & $82.26 \%$ & $88.26 \%$ & $87.94 \%$ & $84.62 \%$ \\
Corresponding moment order & 3 & 11 & 16 & 25 \\
\hline
\end{tabular}

ments used to represent a given image, the MEP method. This method requires no a priori image information.

The processing of the image by blocks of size $(4 \times 4)$, $(8 \times 8),(16 \times 16)$, and $(32 \times 32)$ involves a dramatic reduction of the reconstruction error, and a considerable gain in the computation time, compared with the GRM, in the case of $(128 \times 128)$ binary and multi-gray-level images. Hence we can obtain better reconstruction quality on each block with order of moments considerably low. This new method can involve a blocking artifact, especially at lower reconstruction orders. We propose a new approach for alleviating the blocking artifact by using LBBRM. This method results in significant objective and subjective improvement in the reconstructed image quality, as shown in the experimental results in the case of binary and multi-gray-level images.

The proposed approach which is a combination of the LBBRM with the PME as selection criteria allows not only improvement of the reconstructed images quality but also a surprising acceleration of the reconstruction process, as shown in the results of computation time in Table 3. 


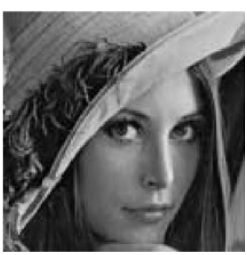

(a)

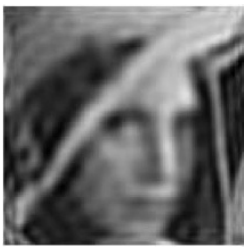

(e)

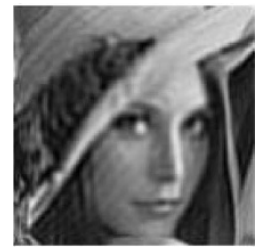

(i)

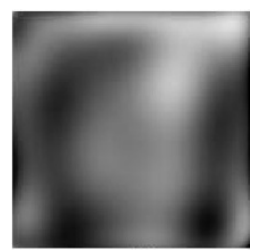

(b)

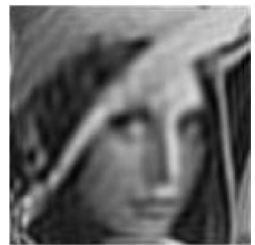

(f)

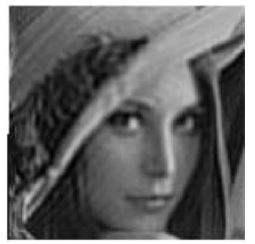

(j)

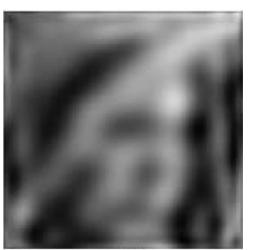

(c)

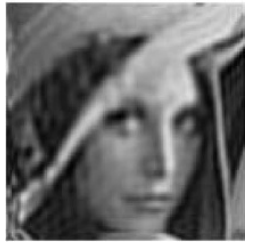

(g)

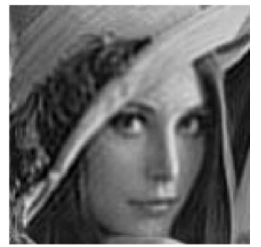

(k)

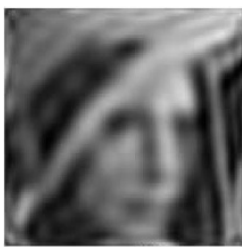

(d)

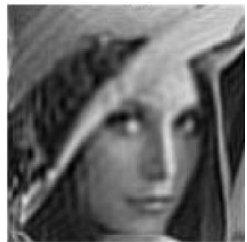

(h)

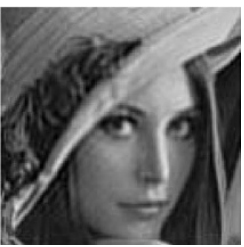

(1)

FIGURE 9: Global reconstruction of the Lena image by the Legendre moments. (a) Original input image; reconstructed images from (b) to (1) represent orders $10,20,30,40,50,60,70,80,90,100$, and 110, respectively.

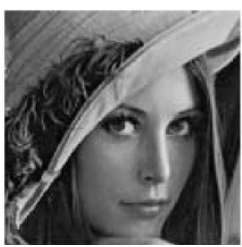

(a)

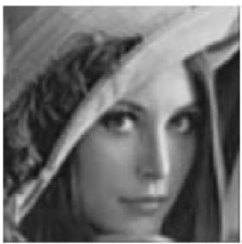

(e)

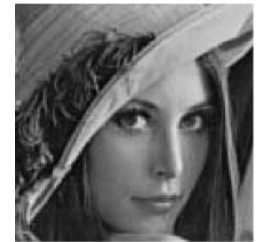

(i)

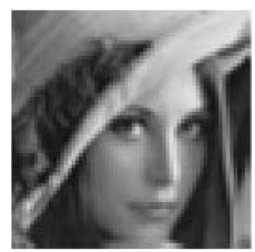

(b)

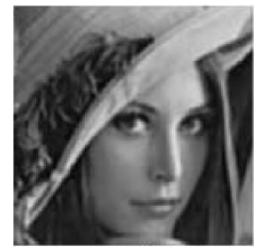

(f)

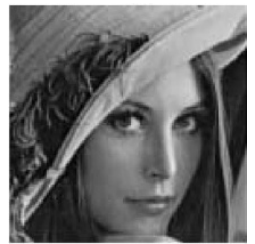

(j)

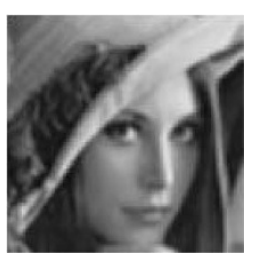

(c)

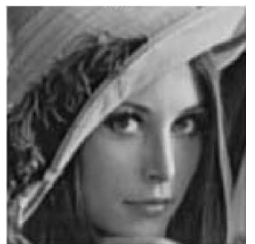

(g)

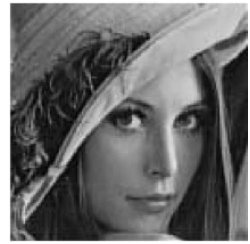

(k)

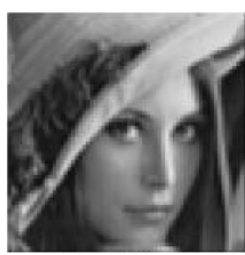

(d)

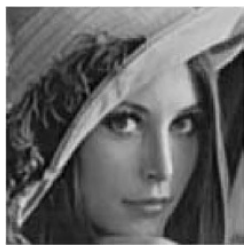

(h)

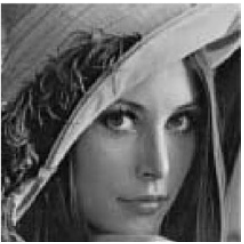

(1)

FIGURE 10: The original Lena image (a), and its reconstructed patterns via the proposed LBBRM using Legendre moments, with the blocks size $(4 \times 4)$; the reconstructed images from (b) to (l) represent orders from 0 to 10 , respectively. 


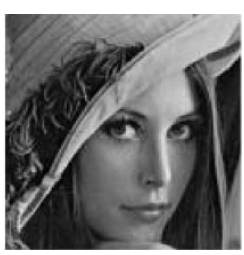

(a)

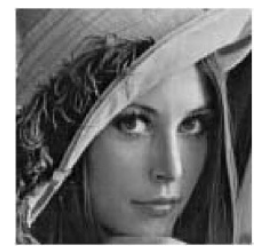

(e)

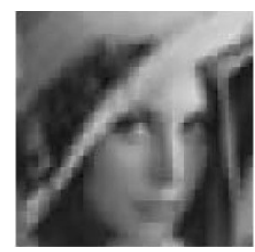

(b)

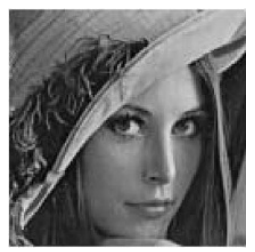

(f)

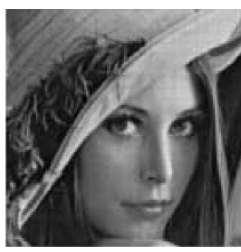

(c)

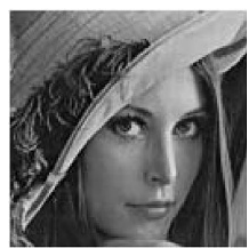

(g)

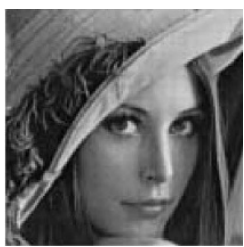

(d)

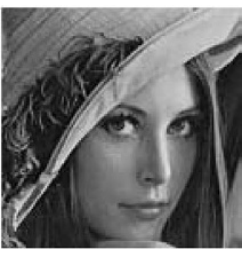

(h)

FIGURE 11: The original Lena image (a) and its reconstructed patterns via the proposed LBBRM with the block size $(8 \times 8)$ using Legendre moments; from (b) to (h) are the reconstructed images for orders 1, 10, 15, 20, 25, 30, and 35, respectively.

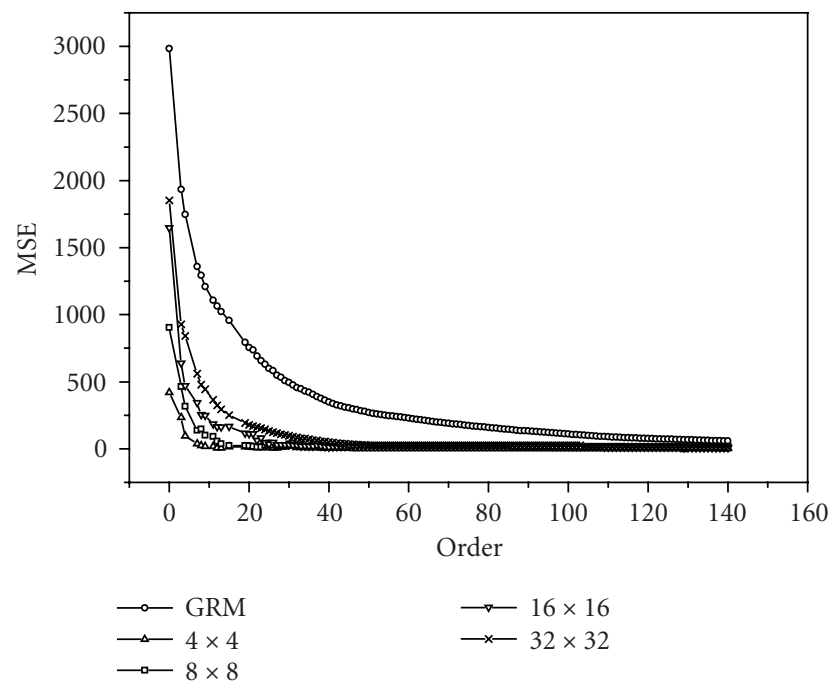

FIGURE 12: Comparison of LBBRM, with different block sizes, and GRM in terms of the reconstruction MSE for Lena image.

\section{REFERENCES}

[1] F. L. Alt, "Digital pattern recognition by moments," Journal of the Association for Computing Machinery, vol. 9, no. 2, pp. 240-258, 1962.

[2] M. K. Hu, "Pattern recognition by moment invariants," Proc. IRE, vol. 49, pp. 1428, September 1961.

[3] M. K. Hu, "Visual pattern recognition by moment invariants," IRE Transactions on Information Theory, vol. 8, no. 2, pp. 179187, 1962.

[4] R. J. Prokop and A. P. Reeves, "A survey of moment-based techniques for unoccluded object representation and recognition," Graphical Models and Image Processing, vol. 54, no. 5, pp. 438-460, 1992.
[5] C.-H. Teh and R. T. Chin, "On digital approximation of moment invariants," Computer Vision, Graphics, and Image Processing, vol. 33, no. 3, pp. 318-326, 1986.

[6] C.-H. Teh and R. T. Chin, "On image analysis by the methods of moments," IEEE Trans. on Pattern Analysis and Machine Intelligence, vol. 10, no. 4, pp. 496-513, 1988.

[7] S. X. Liao and M. Pawlak, "On image analysis by moments," IEEE Trans. on Pattern Analysis and Machine Intelligence, vol. 18, no. 3, pp. 254-266, 1996.

[8] J. Baranger, Analyse numerique, Hermann, Editeurs des Sciences et des Arts, Paris, France, 1991.

[9] S. X. Liao, Image analysis by moments, Ph.D. thesis, Department of Electrical and Computer Engineering, University of Manitoba, Winnipeg, Manitoba, Canada, 1993.

[10] H. Qjidaa and L. Radouane, "Robust line fitting in a noisy image by the method of moments," IEEE Trans. on Pattern Analysis and Machine Intelligence, vol. 21, no. 11, pp. 1216$1223,1999$.

[11] C. Robert, "Modèles statistiques pour l'intelligence artificielle," in Techniques Stochastiques, Masson, Paris, France, 1991.

[12] Y. S. Abu-mostafa and D. Psaltis, "Recognitive aspects of moment invariants," IEEE Trans. on Pattern Analysis and Machine Intelligence, vol. 6, no. 6, pp. 698-706, 1984.

[13] M. Pawlak, "On the reconstruction aspects of moment descriptors," IEEE Transactions on Information Theory, vol. 38, no. 6, pp. 1698-1708, 1992.

[14] M. R. Teague, "Image analysis via the general theory of moments," Journal of Optical Society of America, vol. 70, no. 8, pp. 920-930, 1980.

[15] A. Khotanzad and Y. H. Hong, "Invariant image recognition by Zernike moments," IEEE Trans. on Pattern Analysis and Machine Intelligence, vol. 12, no. 5, pp. 489-497, 1990.

[16] S. X. Liao and M. Pawlak, "On the accuracy of Zernike moments for image analysis," IEEE Trans. on Pattern Analysis and Machine Intelligence, vol. 20, no. 12, pp. 1358-1364, 1998.

[17] X. Zhuang, R. M. Haralick, and Y. Zhao, "Maximum entropy image reconstruction," IEEE Trans. Signal Processing, vol. 39, no. 6, pp. 1478-1480, 1991. 
[18] H. S. Malvar, "The LOT: transform coding without blocking effects," IEEE Trans. Acoustics, Speech, and Signal Processing, vol. 37, no. 4, pp. 553-559, 1989.

[19] A. K. Jain, Fundamentals of Digital Image Processing, PrenticeHall Information and System Sciences Series. Prentice-Hall, Englewood Cliffs, NJ, USA, 1989.

Hakim El Fadili was born in Azrou, Morocco in 1976. He graduated from the Faculty of Science, University Sidi Mohamed Ben Abdellah, Fez, and received the DESA degree in automatic and system analysis in 2000. Presently, he is preparing his Doctorat National in image processing, pattern recognition, and machine intelligence.

Khalid Zenkouar was born in Meknes, Morocco in 1971. He obtained his DUT degree in Mechanic and Computer-Integrated Manufacturing from High school of technology, Fez in 1993. He graduated from the Faculty of Science, University Sidi Mohamed Ben Abdellah, Fez, and received the DESA degree in automatic and system analysis in 2000. Presently, he is preparing his Doctorat National in image processing and mathematical morphology.

Hassan Qjidaa was born in Rabat, Morocco in 1958. He graduated from the Faculty of Science, University Sidi Mohamed Ben Abdellah, Fez, and received the Thèse d'Etat degree in automatic and system analysis in 2000. Presently, he is a Professor of informatics and image processing. He is a member in the Laboratoire d'Electronique, Signaux Systèmes et d'Informatique (LESSI). His current research interests are in image
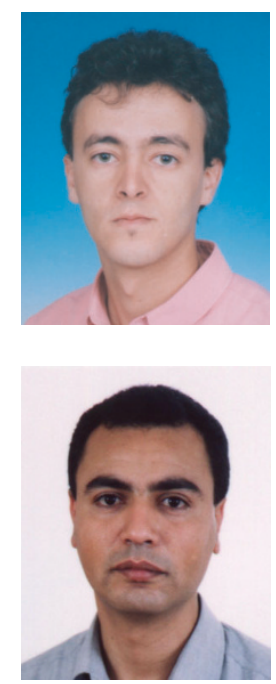
processing, pattern recognition, data analysis, and machine intelligence. 